\title{
Impact of Particles Size Distribution on Diazepam 5mg Tablets Dissolution
}

\author{
B. Pilipović, M. Vehabović, E. Hadžović, N. Šarić
}

Bosnalijek d.d. Jukićeva 53, Sarajevo, Bosnia and Herzegovina

E-mail: berina.p@bosnalijek.ba (B. Pilipović)

Sci Pharm. 2010; 78: 608

doi:10.3797/scipharm.cespt.8.PDD19

Diazepam is one of most frequently prescribed benzodiazepines. It is classified as a class I substance according to the Biopharmaceutics Classification Scheme (BCS). Diazepam tablets were prepared using different API rawmaterials. Before the formulation, we measured particle size distribution on Malvern Instruments Ltd Mastersizer 2000. The tablets were prepared by employing conventional wet granulation. The formulations were evaluated for hardness, friability, content uniformity, in vitro disintegration time (DT), release profiles. The dissolution profile testing is performed on the two formulations with different particles size distribution.

Test parameters were: medium $0.1 \mathrm{~mol} / \mathrm{l}$ hydrochloric acid, $900 \mathrm{ml}$, temperature $37^{\circ} \mathrm{C} \pm 0.5^{\circ} \mathrm{C}$, Mixing Speed $100 \mathrm{rpm}$, Number of tested tablets 12 , Testing Cycle 10, 15, 20, 25 and 30 min. Instrument: Varian Van Kel VK7025, Varian Cary 50. We used the spectrophotometric method with absorbance measuring on $242 \mathrm{~nm}$ for quantification.

Results for particle size distribution for first Diazepam API particle distribution were d (0.10): $88.743 \mu \mathrm{m}$; d (0.50): $305.231 \mu \mathrm{m}$; $d$ (0.90): $655.911 \mu \mathrm{m}$, and for the second raw-material of API was $d(0.10): 1.998 \mu \mathrm{m} ; \mathrm{d}(0.50): 4.419 \mu \mathrm{m}$; $d$ (0.90): $8.845 \mu \mathrm{m}$. The results of diazepam dissolution for two formulations and f2 value with reference drug are presented in Table 1:

Tab. 1.

\begin{tabular}{lrrrrrrrrrr}
\hline & \multicolumn{1}{c}{ Formulation A } & \multicolumn{1}{c}{ Formulation B } \\
minutes & \multicolumn{1}{c}{10} & \multicolumn{1}{c}{15} & \multicolumn{1}{c}{ - } & \multicolumn{1}{c}{ 25 } & \multicolumn{1}{c}{30} & \multicolumn{1}{c}{10} & \multicolumn{1}{c}{15} & \multicolumn{1}{c}{0} & \multicolumn{1}{c}{25} & \multicolumn{1}{c}{30} \\
\hline S.V. & 85.34 & 99.05 & 100.79 & 100.39 & 99.88 & 99,08 & 98,49 & 97,90 & 97,36 & 96,89 \\
SD & 4.65 & 2.57 & 3.20 & 3.34 & 3.31 & 1,32 & 1,32 & 1,28 & 1,27 & 1,28 \\
RSD & 5.45 & 2.60 & 3.17 & 3.32 & 3.31 & 1,33 & 1,34 & 1,31 & 1,31 & 1,32 \\
f2 & & & & & 68,15 & & & & & 99,44
\end{tabular}

It was concluded on the basis of formulation tests that Formulation $B$, for which micronized API was used, has a similar dissolution profile for API as compared to the reference medicine $(\mathfrak{f} 2=99.94)$ in comparison to Formulation $A(\mathfrak{f} 2=68.15)$

[1] George Wong, Charles C. Collins. Pages from Preformulation in Solid Dosage Form Development: Dissolution testing: Informa Healthcare USA, Inc. 2008; 44: p.477-555.

[2] The United States Pharmacopeia.(2007). 30th revision, United States Pharmacopeial Convention, Rockville, MD, USA. 